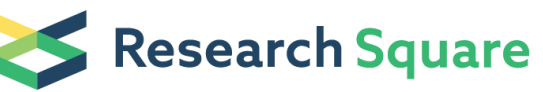

\section{Detection of SARS-CoV-2 in Exhaled Breath from Non-Hospitalized COVID-19-Infected Individuals: A Proof-of-Concept Study}

Cæcilie Leding ( $\nabla$ caecilie.leding@regionh.dk)

Center of Research \& Disruption of Infectious Diseases, Department of Infectious Diseases, Copenhagen University Hospital - Amager and Hvidovre, Hvidovre, Denmark

Julia Skov

AeroCollect, FORCE Technology, Hørsholm, Denmark

Katrine Uhrbrand

AeroCollect, FORCE Technology, Hørsholm, Denmark

Jan Gorm Lisby

Department of Clinical Microbiology, Copenhagen University Hospital - Amager and Hvidovre, Hvidovre, Denmark

\section{Katrine Pedersbæk Hansen}

Center of Research \& Disruption of Infectious Diseases, Department of Infectious Diseases, Copenhagen University Hospital - Amager and Hvidovre, Hvidovre, Denmark

\section{Thomas Benfield}

Center of Research \& Disruption of Infectious Diseases, Department of Infectious Diseases, Copenhagen University Hospital - Amager and Hvidovre, Hvidovre, Denmark

\section{Louise Katrine Duncan}

AeroCollect, FORCE Technology, Hørsholm, Denmark

\section{Research Article}

Keywords: COVID-19, SARS-CoV-2, oropharyngeal

Posted Date: December 16th, 2021

DOI: https://doi.org/10.21203/rs.3.rs-1066707/v1

License: (1) This work is licensed under a Creative Commons Attribution 4.0 International License. Read Full License 


\section{Abstract}

Background: The diagnosis of COVID-19 is based on detection of SARS-CoV-2 in oro-/nasopharyngel swabs, but because of uncomfortness and minor risk for the tested individual during the swab procedure, detection of SARS-CoV-2 has been investigated in other biological matrixes.

Methods: In this proof-of-concept study, confirmed SARS-CoV-2-infected individuals performed a daily air sample through five days. Air samples were obtained through a non-invasive electrostatic air sampler. Detection of SARS-CoV-2 RNA were determined with qRT-PCR. The association of positive samples with clinical characteristics was evaluated through mixed-effect models.

Results: We obtained 665 air samples from 111 included patients with confirmed SARS-CoV-2 infection. Overall, 52 individuals (46.8\%) had at least one positive air sample, and 129 (19.4\%) air samples were positive for SARS-CoV-2. Patients with symptoms or a symptom duration $\leq$ four days had significantly higher odds of having a positive air sample. Cycle threshold values were significantly lower in samples obtained $\leq 4$ days from symptom onset. Neither variant of SARS-CoV-2 nor method of air sampling were associated with a positive air sample.

Conclusion: We demonstrate that SARS-CoV-2 is detectable in human breath, and suggest further evaluation of air sampling considering the non-invasive, point-of-care method.

\section{Introduction}

The gold standard in the diagnosis of COVID-19 is detection of SARS-CoV-2 genomes from naso- or oropharyngeal (OP) swabs using real-time reverse transcription polymerase chain reaction (RT-PCR). However, the procedure of obtaining material from the upper respiratory tract is invasive and thus uncomfortable and with minor risk for the tested individual. Furthermore, it requires trained staff that risk infection following virus exposure during the swabbing procedure. Therefore, detection of SARS-CoV-2 based on other sample materials have been investigated including plasma, serum, urine, and feces ${ }^{1}$. It is well-known that the virus, among other routes, transmits through aerosols and droplets from infected individuals. This has given the rationale of investigating exhaled breath as an alternative specimen type in the diagnosis of COVID-19 as well as a focus on the strategy to mitigate the transmission of SARSCoV- $2^{2-4}$. Air sampling of exhaled breath could provide an easy non-invasive testing alternative with potential for self-sampling. Furthermore, studying the emission of SARS-CoV-2 in exhaled breath of infected individuals can provide valuable information e.g. in relation to identifying high risk exposure activities.

A previous study established that SARS-CoV-2 was detectable in air close to the snouts of minks using an electrostatic air sampler (AeroCollect ${ }^{\circledR}$, FORCE Technology, Hørsholm, Denmark) ${ }^{5}$. The aim of the current proof-of-concept study was to investigate whether the AeroCollect ${ }^{\circledR}$ device was capable of detecting SARS-CoV-2 in exhaled breath from humans and to investigate if the detection rate differed by duration of symptoms, the variant of SARS-CoV-2 and method of air sampling. 


\section{Material And Methods \\ Study cohorts}

This prospective, observational proof-of-concept study (cohort 1) was performed at Amager-Hvidovre Hospital, Copenhagen, Denmark, between December 2020 and May 2021.

Individuals with recently nucleic acid amplification test (NAAT) confirmed SARS-CoV-2 positivity were identified through the Department of Clinical Microbiology. Eligible participants (not hospitalized and $\geq$ 18 years) and their household members (not hospitalized and $\geq 12$ years) were invited to participate in the study. Prior to study entry, all participants provided written informed consent. The study was approved by the Regional Data Protection Centre (record no. P-2020-1219). The study was exempted from ethical approval according to the Regional Ethics Committee of the Capital Region of Denmark (record no. $\mathrm{H}$ 20020554). The decision was made because the committee assessed that the aim of the current study was to achieve knowledge about SARS-CoV-2 in the environment and not about human biology. All methods were performed in accordance with the relevant guidelines and regulations.

Oropharyngeal swabs for NAAT was obtained from household members at day one and five. The swabs were analyzed at the Department of Clinical Microbiology and included sequencing of SARS-CoV-2 if the virus was detected. Clinical information at baseline from each participant and information of symptoms of SARS-CoV-2 from day one to five were provided in a questionnaire and registered in the REDCap database.

Cohort 2 consisted of hospitalized individuals with confirmed SARS-CoV-2 infection. These individuals were included in March 2020.

\section{Sample collection}

Sampling of aerosolized SARS-CoV-2 was carried out using the AeroCollect $\circledast$ air sampling technology. The AeroCollect ${ }^{\circledR}$ air sampler was mounted with chip-based sample chamber and operated at a flowrate of 0.2 liter per minute. During the sampling, viruses present in the exhaled air were captured and retained electrostatically in the sample chamber. Particle emission rates of aerosols have been shown to be positively correlated with vocal loudness, and thus are higher in singing and loud talking than during normal talking or breathing ${ }^{6-8}$. Therefore, air samples in this study were obtained from the breathing zone of the participants while singing or talking loudly.

Participants in cohort 1 were requested to provide one air sample per day during five consecutive days. Time of air sampling were four minutes per sample. In order to evaluate air sampling methods, a subset of participants in cohort 1 was asked to provide two matched air samples a day for five consecutive days, one singing/loud talking for four minutes and one exhaling air for four minutes, for evaluating methods on air sampling.

Patients in cohort 2 provided one air sample per patient by exhaling for one minute. 
All samples were blind coded and transported within one week of last sampling day to FORCE Technology, Hørsholm, Denmark for analysis.

\section{Detection of SARS-CoV-2 in air samples}

Aerosolized SARS-CoV-2 were eluted from the AeroCollect sample chamber using $25 \mu$ l nuclease-free water, flushing the chamber 15 times. The Agilent AriaMX Real-time PCR system (Agilent, United States) was used for detection of SARS-CoV-2. The detection was performed in duplicates using the ViroType SARS-COV-2 detection assay, consisting of the Virotype Mix +IC(JOE)-RNA reaction mixture (INDICAL BIOSCIENCE, Germany) and the Virotype SARS-CoV-2 (E-Sarbecco) Primers/Probes mix (INDICAL BIOSCIENCE, Germany), targeting the E gene of SARS-CoV-2. The RT-PCR reaction was carried out according the manufacturer's recommendations using eight $\mu \mathrm{l}$ sample template in a total reaction volume of $25 \mu$. Reaction conditions were RT at $50^{\circ} \mathrm{C}$ for 10 minutes, activation at $95^{\circ} \mathrm{C}$ for two minutes followed by 45 cycles of $95^{\circ} \mathrm{C}$ for five seconds and $60^{\circ} \mathrm{C}$ for 30 seconds.

All reactions contained an internal reaction control. In each PCR run, a positive control consisting of the Virotype SARS-CoV-2 (E-Sarbecco) Positive Control (INDICAL BIOSCIENCE, Germany) and a negative template control (nuclease-free water) was included. Fluorescence was measured at the end of each cycle. Baseline corrected raw fluorescence $(\Delta R)$ in the Agilent AriaMX software 1.71 (Agilent) was used for setting the fluorescence thresholds. Signals exceeding this threshold with cycle threshold (Ct) value of 45 or below were considered positive.

Interpretation of RT-PCR results of the air samples were blinded to the clinical information of the participants. Analysis results of the air samples were subsequently sent to a clinician at Hvidovre Hospital who combined the data with the clinical information.

\section{Statistical analysis}

Baseline characteristics are presented as numbers with percentages or medians with an interquartile range (IQR).

To asses any association of positive air samples between groups of participants, we performed generalized linear mixed-effects models. Fixed effects in the models were symptom duration ( $\leq$ four or $>$ four days), symptoms (yes or no), variant of SARS-CoV-2 (wildtype or B.1.1.7) and method of air sampling (singing/loud talking or exhaling air), respectively, corresponding to the subgroups of interest. We used random within person intercept to account for baseline variability between participants in all four models. Furthermore, random intercept of each day of air sampling were included in the model of method of air sampling to account for variability between days.

Difference in $\mathrm{Ct}$ values between the abovementioned subgroups were assessed with linear mixed-effects models. Fixed effects in the models corresponded to the subgroup of interest, and random intercept of each participant were also included. Additionally, random intercept of each day of air sampling were included in the model. 
Estimates of association were presented as odds ratios (OR) with $95 \%$ confidence interval $(95 \% \mathrm{Cl})$. Goodness-of-fit for generalized linear mixed models was evaluated with Hosmer-Lemeshow test.

$P$ values $<0.05$ were considered statistically significant. Data analyses were performed using $\mathrm{R}$ version 3.6.0 (R Foundation for Statistical Computing, Vienna, Austria).

\section{Results}

We recruited 133 individuals of which 22 were excluded (Figure 1) resulting in 111 included subjects with NAAT confirmed SARS-CoV-2. Clinical characteristics of included participants are shown in Table 1. 
Table 1

Characteristics of subjects with confirmed SARS-CoV-2 infection.

\section{Characteristics}

Sex

Female, $\mathrm{n}(\%)$

Male, $\mathrm{n}(\%)$

Age, median [IQR]

Comorbidity

Yes, n (\%)

No, $n(\%)$

SARS-CoV-2 confirmed prior to inclusion

Yes, n (\%)

No, $n(\%)$

Symptoms at inclusion

Yes, $\mathrm{n}(\%)$

No, $n(\%)$

Symptom duration at inclusion, median [IQR]

Missing, $\mathrm{n}(\%)$

Symptoms at any time

Yes, $\mathrm{n}(\%)$

No, $n(\%)$

Missing, $\mathrm{n}(\%)$

SARS-CoV-2 subtype

Wildtype, $\mathrm{n}(\%)$

B.1.1.7, n (\%)

Missing

Full air sampling

Yes, n (\%)

No, $n(\%)$

Abbreviations: n, number; IQR, interquartile range; SARS-CoV-2, severe acute respiratory coronavirus 2 .
Overall $(n=111)$

$66(59.5)$

$45(40.5)$

$40[29,54]$

$33(30.0)$

$77(70.0)$

$106(95.5)$

$5(4.5)$

$100(90.1)$

11 (9.9)

$3[2,5]$

2 (2)

105 (95.5)

5 (4.5)

1

18 (19.8)

73 (80.2)

20

102 (91.9)

9 (8.1)

Page 6/16 
Any positive air sample

Yes, n (\%)

No, $n(\%)$

Abbreviations: $n$, number; IQR, interquartile range; SARS-CoV-2, severe acute respiratory coronavirus 2 .

Of the 111 individuals in the study, $52(46.8 \%)$ had at least one positive air sample. In all, we obtained 665 air samples, 536 during singing/talking loud and 129 during exhaling air, from the NAAT confirmed individuals (Table 2). Of the 665 air samples, 129 (19.4\%) were found positive for SARS-CoV-2.

Table 2

Number of air samples (positive/negative) stratified by subgroup.

\begin{tabular}{|c|c|c|c|}
\hline Subgroup & $\begin{array}{l}\text { Positive air samples, } n \\
\text { (\%) }\end{array}$ & $\begin{array}{l}\text { Negative air samples, } n \\
(\%)\end{array}$ & $\begin{array}{l}\text { Total air samples, } \\
\mathbf{n}\end{array}$ \\
\hline All air samples* & $129(19.4)$ & $536(80.6)$ & 665 \\
\hline \multicolumn{4}{|l|}{ Symptoms** } \\
\hline Symptomatic patients & $95(21.1)$ & 355 (78.9) & 450 \\
\hline Asymptomatic patients & $6(7.2)$ & $77(92.8)$ & 83 \\
\hline \multicolumn{4}{|l|}{ Duration of symptoms ${ }^{\star \star}$} \\
\hline $\begin{array}{l}\text { Symptom duration } \leq 4 \\
\text { days }\end{array}$ & $49(28.0)$ & $126(72.0)$ & 175 \\
\hline $\begin{array}{l}\text { Symptom duration }>4 \\
\text { days }\end{array}$ & $44(14.5)$ & $260(85.5)$ & 304 \\
\hline \multicolumn{4}{|l|}{ Variant of SARS-CoV-2** } \\
\hline Wildtype & $18(20.7)$ & $69(79.3)$ & 87 \\
\hline B.1.1.7. & $78(22.0)$ & $276(78.0)$ & 354 \\
\hline \multicolumn{4}{|l|}{ Method of air sampling } \\
\hline Singing/loud talking & $101(18.8)$ & $435(81.2)$ & 536 \\
\hline Exhaling & $28(21.7)$ & $101(78.3)$ & 129 \\
\hline \multicolumn{4}{|c|}{ Abbreviations: n, number; SARS-CoV-2, severe acute respiratory coronavirus 2 . } \\
\hline \multicolumn{4}{|c|}{ *obtained through singing/loud talking and exhaling } \\
\hline
\end{tabular}




\section{Subgroup analyses}

Figure 2 shows the distribution of air samples (singing/loud talking) obtained according to days from symptom onset. Generalized linear mixed-effects model estimates of having a positive air sample stratified by subgroups are demonstrated in Figure 3. Linear mixed-effect models for $\mathrm{Ct}$ values stratified by subgroups are presented in Table 3 .

Table 3

Linear mixed-effect model analyses for Ct values stratified by subgroups.

\begin{tabular}{|llll|}
\hline Subgroup & $\begin{array}{l}\text { Mean Ct value } \\
{[95 \% \mathrm{Cl}]}\end{array}$ & $\begin{array}{l}\text { Mean difference of } \\
\text { Ct value [95\% Cl] }\end{array}$ & P value \\
\hline Symptoms & & & \\
\hline Asymptomatic patients & $37.17[34.75 ; 39.60]$ & Ref & 0.267 \\
\hline Symptomatic patients & $35.77[35.16 ; 36.38]$ & $-1.40[-3.90 ; 1.09]$ & \\
\hline Duration of symptoms & & & 0.002 \\
\hline Symptom duration > 4 days & $36.74[35.88 ; 37.59]$ & Ref & \\
\hline Symptom duration $\leq 4$ days & $34.87[34.08 ; 35.68]$ & $-1.86[-3.05 ;-0.68]$ & 0.894 \\
\hline $\begin{array}{l}\text { Variant of SARS-CoV-2 } \\
\text { Wildtype }\end{array}$ & $35.80[34.43 ; 37.18]$ & Ref & \\
\hline B.1.1.7. & $35.71[35.05 ; 36.36]$ & $-0.10[-1.63 ; 1.42]$ & \\
\hline Method of air sampling & & & 0.545 \\
\hline Exhaling & $35.54[34.22 ; 36.86]$ & Ref & \\
\hline Singing/loud talking & $35.92[35.02 ; 36.83]$ & $0.38[-0.90 ; 1.67]$ & \\
\hline $\begin{array}{l}\text { Abbreviations: Ct, cycle threshold; Cl, confidence interval; IQR, interquartile range; Ref, reference; SARS- } \\
\text { CoV-2, severe acute respiratory coronavirus 2. }\end{array}$ & & \\
\hline *of samples obtained through singing/loud talking & & \\
\hline
\end{tabular}

Individuals with symptoms at time of air sampling had significantly higher odds of having a positive air sample compared to asymptomatic individuals $(\mathrm{OR}, 4.4 ; \mathrm{p}=0.017)$. The mean difference of $\mathrm{Ct}$ values between symptomatic and asymptomatic patients was not significant $(P=0.267)$.

Odds of having a positive air sample among participants with short duration of symptoms ( $\leq$ four days) compared to longer symptom duration ( $>$ four days) were significantly higher $(O R, 2.2, p=0.015)$. Cycle threshold values of air samples from participants with shorter duration of symptoms were significantly lower compared to samples obtained from participants with longer symptom duration $(p=0.002)$. 
We found no difference in neither OR of having a positive sample nor Ct values between groups of SARSCoV-2 variant (B.1.1.7 or wildtype) or method of air sampling (singing/loud talking or exhaling), respectively.

When evaluating assumptions of linear mixed-effect models, some models did not have normal distributed residuals. Therefore, secondary models excluding measurements deviating from the normal assumption was fitted for comparison. None of the secondary models let to different interpretation of the results, why results from the original models are presented.

\section{Hospitalized participants}

Nine patients hospitalized with COVID-19 were included in March 2020. Days from symptom onset were between seven and 17 at time of air sampling. No air samples from these hospitalized patients were positive.

\section{Discussion}

In this proof-of-concept study, we demonstrate that SARS-CoV-2 can be identified in exhaled breath from humans. This is the first study to evaluate SARS-CoV-2 in exhaled breath over five consecutive days in more than 100 confirmed SARS-CoV-2 patients providing more than 650 air samples.

Different studies have investigated exhaled breath as a biological matrix in the diagnosis of SARS-CoV$2^{9-14}$. The diversity of devices and techniques is wide which is reflected in the varying detection rates $(11.1 \%-70.0 \%)$ of these studies. We found an overall detection rate of $19.4 \%$ when including all analyzed air samples, which were obtained from one day before through 17 days after symptom onset. The detection rate increased to $28.0 \%$ in earlier stages of the disease, i.e. in samples obtained $\leq$ four days from symptom onset. When taking individual variability of each patient into account, the odds of having a positive sample were 2.2 times higher in air samples obtained $\leq$ four days from symptom onset compared to > four days. This corresponds to the significantly lower $\mathrm{Ct}$ value observed in these samples compared to $\mathrm{Ct}$ values in samples obtained in later stages of the disease. Furthermore, air samples obtained from the second cohort with hospitalized patients with symptoms for at least seven days were all negative. In previous papers, viral load in nose and/or throat swabs has been shown to peak on or soon after the day of symptom onset and then deteriorate, which is in accordance with our results ${ }^{15-17}$. Other papers using air sampling for detection of SARS-CoV-2 have also reported higher detection rates and/or lower $\mathrm{Ct}$ values with shorter symptom duration ${ }^{10,11,14}$.

Patients with symptoms at time of air sampling had 4.4 higher odds of having a SARS-CoV-2 positive air sample compared to patients without symptoms. Previous studies evaluating exhaled air samples from COVID-19 patients only report on symptomatic patients why they are not directly comparable with our results. Although a higher proportion of positive samples from symptomatic participants, no difference in $\mathrm{Ct}$ value between symptomatic compared to asymptomatic participants were found in our study. Knowledge on viral load in a- and presymptomatic SARS-CoV-2-infected individuals is scarce, but few 
studies suggested that viral load in naso-/oropharyngeal swabs from a- or pre-symptomatic individuals did not differ from viral load in symptomatic individuals ${ }^{18}$. In our study, only six (7.2\%) air samples from asymptomatic individuals turned out positive, providing a wide IQR for Ct value reflecting a too small sample size of this group. Therefore, this result must be interpreted with caution.

A more transmittable lineage of SARS-CoV-2 (Alpha or B.1.1.7) was rapidly spread in December 2020 in the UK and later to other countries ${ }^{19,20}$. A study reported lower $\mathrm{Ct}$ values (i.e. higher viral load) in upper respiratory material from patients infected with B.1.1.7 compared to those infected with previous lineages ${ }^{21}$. We did not find a significant difference in neither $\mathrm{OR}$ og positive samples nor $\mathrm{Ct}$ value between air samples taken from B.1.1.7-infected individuals compared with wildtype-infected individuals.

The strength of this study is the large sample size and large number of air samples. Furthermore, our findings are in accordance with the literature, reporting higher detection rates in earlier disease stages. Despite the potential observed using air sampling for detection of SARS-CoV-2 in the early phase of the infection, our results suggest that there are still room for optimization of the method, as our overall detection rate was relatively low compared to other studies on air sampling. This could partly be explained by the fact that we performed RT-PCR on the air samples targeting only the E gene. Ryan et al. reported a mean detection rate of SARS-CoV-2 in air samples of $68 \%$ using two targets (E and S) which increased to $93.5 \%$ when RT-PCR were performed using four gene targets (S/E/N/Orf1ab) ${ }^{9}$. This indicates that analyzing several genes could increase the overall detection rate. Additionally, our detection rate of positive air samples was low with relatively high Ct values compared with oro-/nasopharyngeal swab testing, and the present study do not suggest air sampling as an alternative diagnostic method to standard oro-/nasopharyngeal swab for COVID-19. However, not all virus particles present in cell material from pharynx necessarily aerosolize, why assessing SARS-CoV-2 in exhaled breath could reflect the infectiousness more appropriate than naso-/oropharyngeal swabs. Considering this and the non-invasive method, the potential of detecting the virus in this biological material is worth exploring. With further optimization of air sampling, this method could play a role in population-based monitoring of SARS-CoV2 as an early warning system.

In conclusion, we demonstrate that SARS-CoV-2 is detectable in humans using the air sampling device AeroCollect ${ }^{\circledR}$ and that odds of having a positive air sample was significantly higher in earlier disease stages, and in symptomatic individuals compared to asymptomatic individuals. Further investigation on air sampling as a method for detecting SARS-CoV-2 and studying emission and transmission is required.

\section{Declarations}

\section{Data Availability}

The datasets generated during and/or analysed during the current study are available from the corresponding author on reasonable request.

\section{Acknowledgments}

Page 10/16 
We wish to thank Karsten Brandt Andersen from AeroCollect, FORCE Technology, for method development.

\section{Author contributions}

Concept and design: C.L., J.S., T.B. and L.K.D.

Acquisition, analysis and interpretation of data: all authors

Drafting the article or revising it critically for important intellectual content: all authors

Final approval of the version to be published: all authors

\section{Funding}

This work have been financed by The Danish Agency for Higher Education and Science.

\section{Competing interests}

J.S., K.U. and L.K.D. report employment of AeroCollect, FORCE Technology, Hørsholm, Denmark.

All other authors report no potential conflicts.

\section{References}

1. Shenoy, S. SARS-CoV-2 (COVID-19), viral load and clinical outcomes; lessons learned one year into the pandemic: A systematic review. World J. Crit. Care Med. <background-color:\#FFCC66;bverticalalign:super;>10</background-color:\#FFCC66;bvertical-align:super;>, 132-150 (2021).

2. Tang, S. et al. Aerosol transmission of SARS-CoV-2? Evidence, prevention and control. Environ. Int. <background-color:\#FFCC66;bvertical-align:super;>144</background-color:\#FFCC66;bverticalalign:super;>, 106039 (2020).

3. Morawska, L. \& Cao, J. Airborne transmission of SARS-CoV-2: The world should face the reality. Environ. Int. <background-color:\#FFCC66;bvertical-align:super;>139</backgroundcolor:\#FFCC66;bvertical-align:super;>, 105730 (2020).

4. van Doremalen, N. et al. Aerosol and Surface Stability of SARS-CoV-2 as Compared with SARS-CoV1. N. Engl. J. Med. NEJMc2004973 (2020) doi:10.1056/NEJMc2004973.

5. Boklund, A. et al. SARS-CoV-2 in Danish Mink Farms: Course of the Epidemic and a Descriptive Analysis of the Outbreaks in 2020. Anim. Open Access J. MDPI<backgroundcolor:\#FFCC66;bvertical-align:super;>11</background-color:\#FFCC66;bvertical-align:super;>, (2021).

6. Alsved, M. et al. Exhaled respiratory particles during singing and talking. Aerosol Sci. Technol. <background-color:\#FFCC66;bvertical-align:super;>54</background-color:\#FFCC66;bverticalalign:super;>, 1245-1248 (2020). 
7. Mürbe, D., Kriegel, M., Lange, J., Rotheudt, H. \& Fleischer, M. Aerosol emission in professional singing of classical music. Sci. Rep. <background-color:\#FFCC66;bvertical-align:super;>11</backgroundcolor:\#FFCC66;bvertical-align:super;>, 14861-14861 (2021).

8. Asadi, S. et al. Aerosol emission and superemission during human speech increase with voice loudness. Sci. Rep. <background-color:\#FFCC66;bvertical-align:super;>9</backgroundcolor:\#FFCC66;bvertical-align:super;>, (2019).

9. Ryan, D. J. et al. Use of exhaled breath condensate (EBC) in the diagnosis of SARS-COV-2 (COVID19). Thorax thoraxjnl-2020-215705 (2020) doi:10.1136/thoraxjnl-2020-215705.

10. Sawano, M., Takeshita, K., Ohno, H. \& Oka, H. RT-PCR diagnosis of COVID-19 from exhaled breath condensate: a clinical study. J. Breath Res. <background-color:\#FFCC66;bverticalalign:super;>15</background-color:\#FFCC66;bvertical-align:super;>, (2021).

11. Ma, J. et al. Coronavirus Disease 2019 Patients in Earlier Stages Exhaled Millions of Severe Acute Respiratory Syndrome Coronavirus 2 Per Hour. Clin. Infect. Dis. <backgroundcolor:\#FFCC66;bvertical-align:super;>72</background-color:\#FFCC66;bvertical-align:super;>, e652e654 (2021).

12. Zhou, L. et al. Breath-, air- and surface-borne SARS-CoV-2 in hospitals. J. Aerosol Sci. <backgroundcolor:\#FFCC66;bvertical-align:super;>152</background-color:\#FFCC66;bvertical-align:super;>, 105693 (2021).

13. Li, X. et al. Detecting SARS-CoV-2 in the Breath of COVID-19 Patients. Front. Med. <backgroundcolor:\#FFCC66;bvertical-align:super;>8</background-color:\#FFCC66;bvertical-align:super;>, (2021).

14. Malik, M., Kunze, A.-C., Bahmer, T., Herget-Rosenthal, S. \& Kunze, T. SARS-CoV-2: Viral Loads of Exhaled Breath and Oronasopharyngeal Specimens in Hospitalized Patients with COVID-19. Int. J. Infect. Dis. IJID Off. Publ. Int. Soc. Infect. Dis. <background-color:\#FFCC66;bverticalalign:super;>110</background-color:\#FFCC66;bvertical-align:super;>, 105-110 (2021).

15. Wölfel, R. et al. Virological assessment of hospitalized patients with COVID-2019. Nature <background-color:\#FFCC66;bvertical-align:super;>581</background-color:\#FFCC66;bverticalalign:super;>, 465-469 (2020).

16. Zou, L. et al. SARS-CoV-2 Viral Load in Upper Respiratory Specimens of Infected Patients. N. Engl. J. Med. <background-color:\#FFCC66;bvertical-align:super;>382</background-color:\#FFCC66;bverticalalign:super;>, 1177-1179 (2020).

17. He, X. et al. Temporal dynamics in viral shedding and transmissibility of COVID-19. Nat. Med. <background-color:\#FFCC66;bvertical-align:super;>26</background-color:\#FFCC66;bverticalalign:super;>, 672-675 (2020).

18. Byrne, A. W. et al. Inferred duration of infectious period of SARS-CoV-2: rapid scoping review and analysis of available evidence for asymptomatic and symptomatic COVID-19 cases. BMJ Open $<$ background-color:\#FFCC66;bvertical-align:super;>10</background-color:\#FFCC66;bverticalalign:super;>, e039856 (2020). 
19. Davies, N. G. et al. Estimated transmissibility and impact of SARS-CoV-2 lineage B.1.1.7 in England. Science<background-color:\#FFCC66;bvertical-align:super;>372</backgroundcolor:\#FFCC66;bvertical-align:super;>, eabg3055 (2021).

20. Bager, P. et al. Risk of hospitalisation associated with infection with SARS-CoV-2 lineage B.1.1.7 in Denmark: an observational cohort study. Lancet Infect. Dis. (2021) doi:10.1016/S14733099(21)00290-5.

21. Challen, R. et al. Risk of mortality in patients infected with SARS-CoV-2 variant of concern 202012/1: matched cohort study. The BMJ <background-color:\#FFCC66;bverticalalign:super;>372</background-color:\#FFCC66;bvertical-align:super;>, n579 (2021).

\section{Figures}




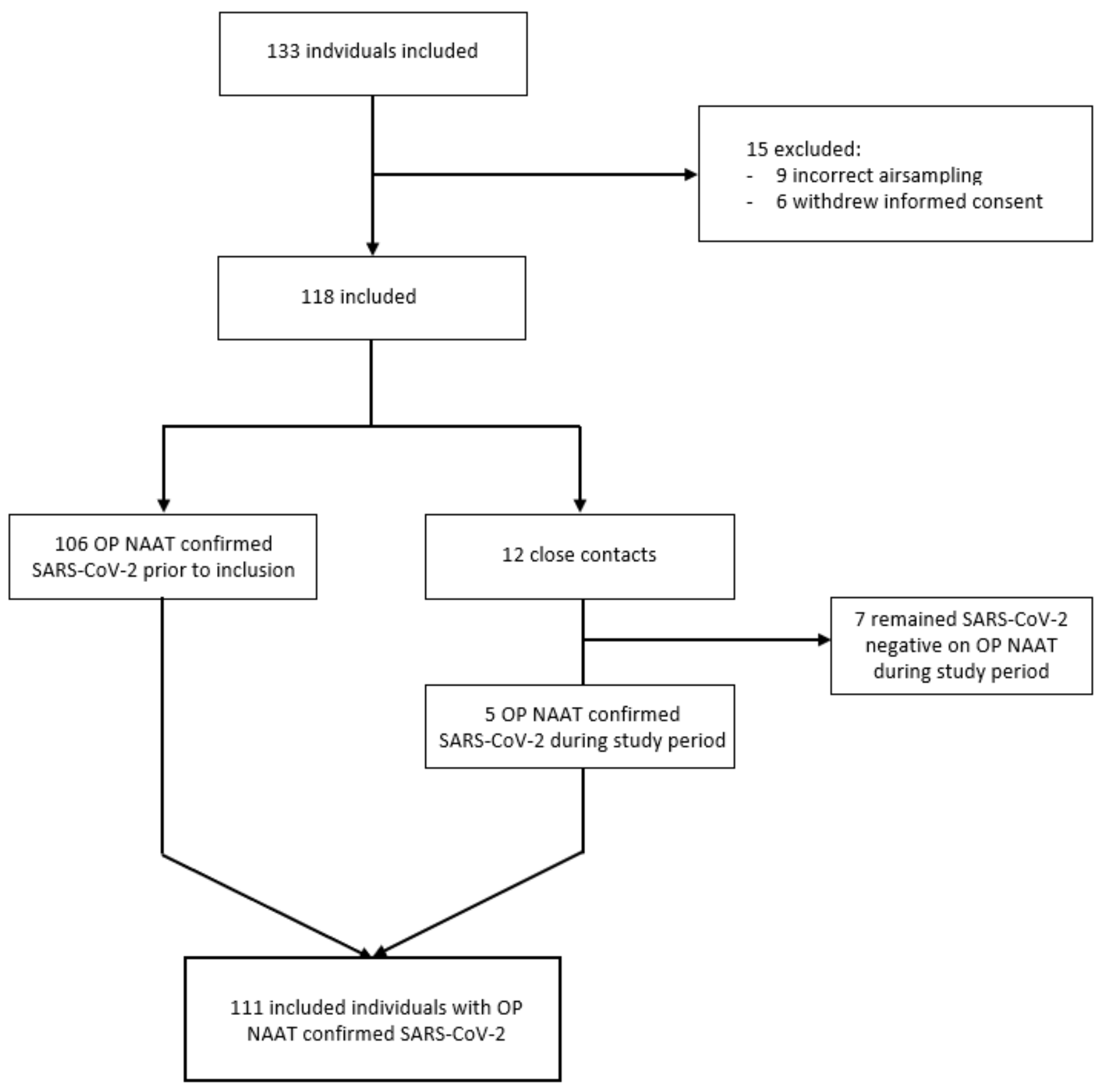

Figure 1

$<\mathrm{p}>$ Flow chart of enrollment of patients. Abbreviations: NAAT, nucleic acid amplification test; OP, oropharyngeal; SARS-CoV-2, severe acute respiratory coronavirus $2 .</ p>$ 


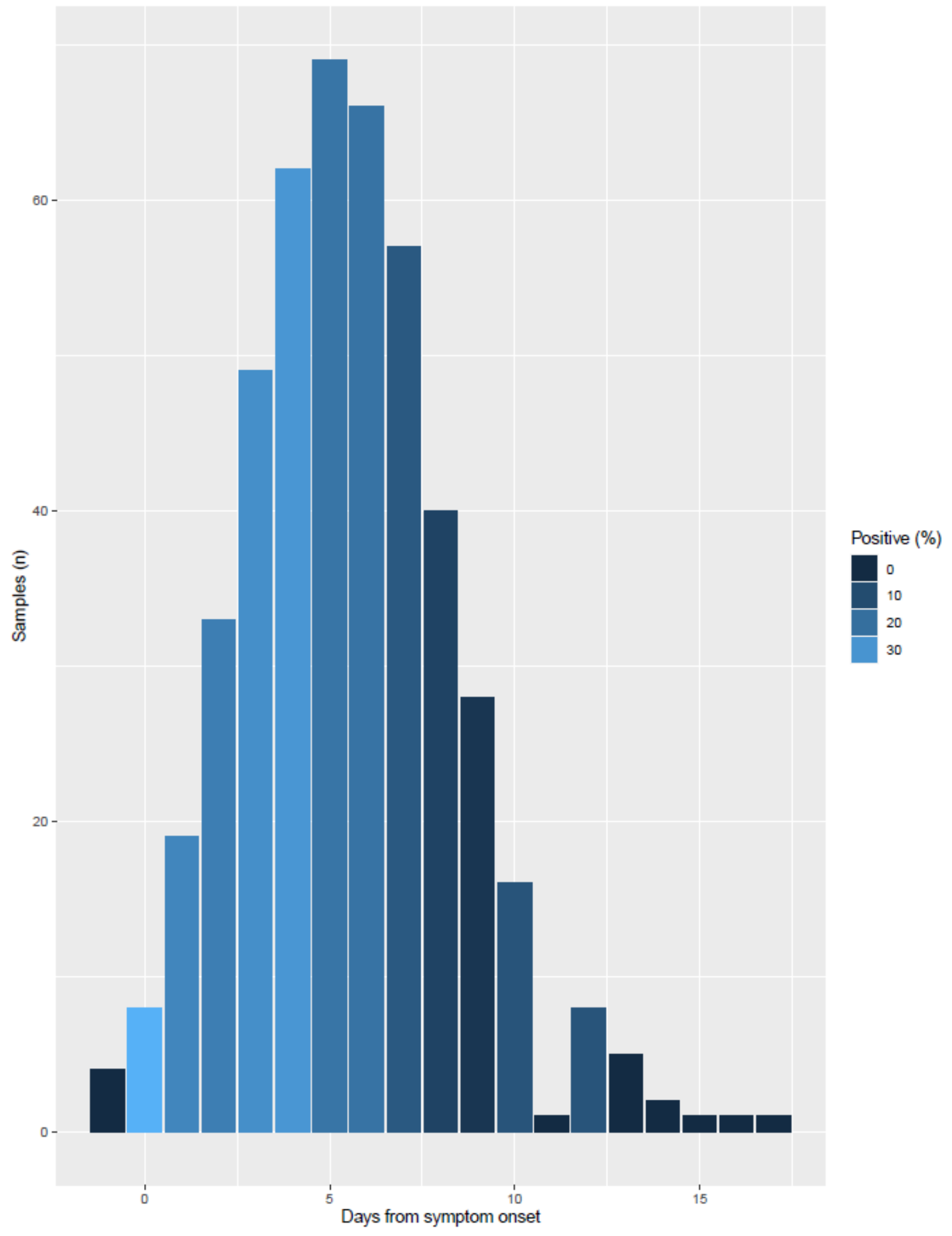

Figure 2

$<p>$ Total number of air samples and percent positive per day from symptom obtained from confirmed COVID-19 patients. $</ p>$ 


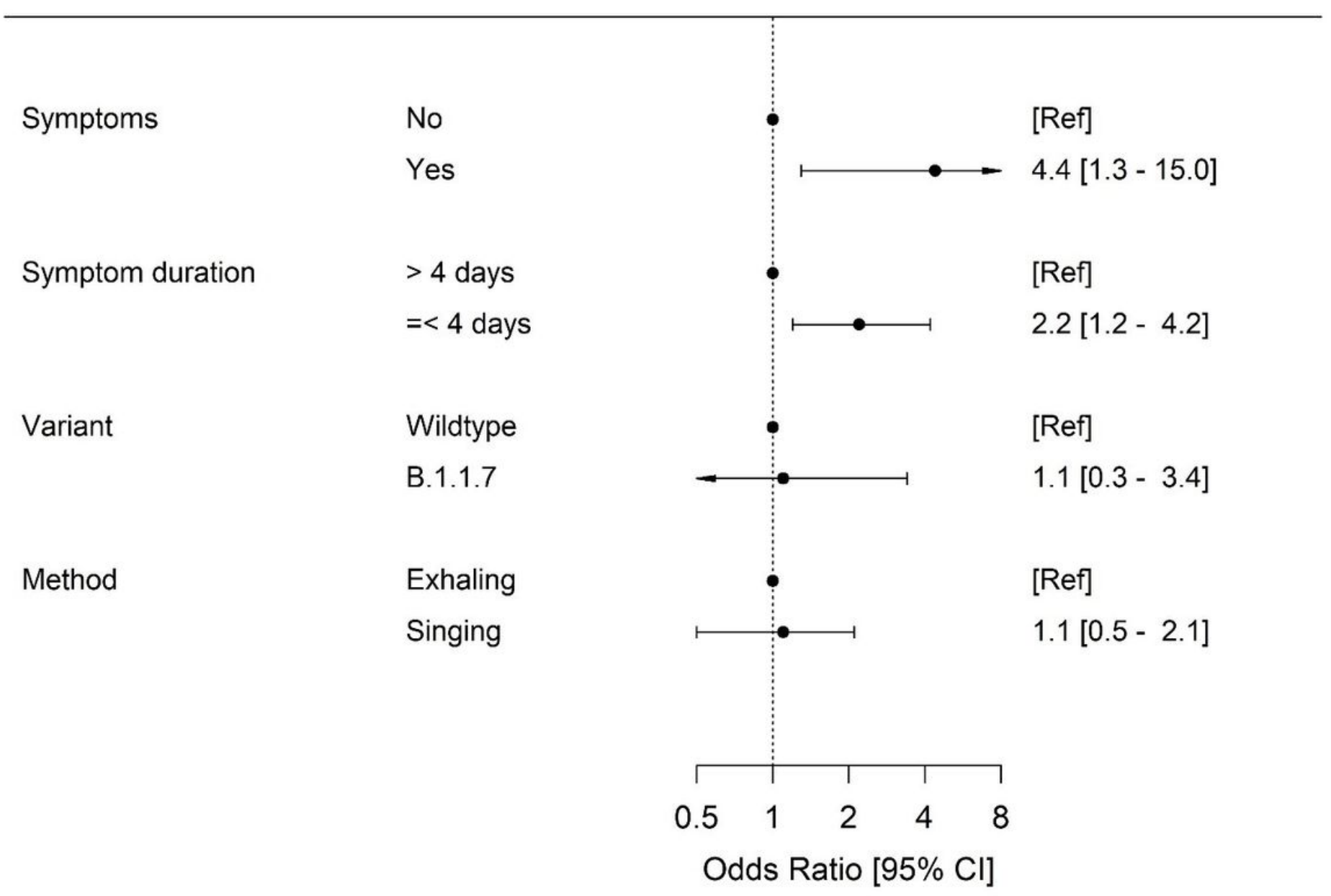

\section{Figure 3}

$<p>$ Generalized linear mixed model analyses of having a positive air sample stratified by subgroups presented as OR. Abbreviations: $\mathrm{Cl}$, confidence interval; OR, odds ratio; ref, reference. $</ p>$ 